\title{
Atendimento em Saúde à Pessoa com Deficiência e a Formação Inicial do Profissional de Saúde: o que Há rnTre Nós?
}

\author{
Health Care for the Person with Disability and the Initial Training of \\ the HeALTh PROfESSIONAL: WHAT IS BETWEEN Us?
}

\author{
Marhla Laiane de Brito ASSUNÇÃO' \\ Francis Natally de Almeida ANACLETO ${ }^{3}$ \\ Ramon MISSIAS-MOREIRA ${ }^{4}$ \\ Albertino José FERREIRA NETO 5 \\ Cheila Nataly Galindo BEDOR ${ }^{6}$
}

\begin{abstract}
RESUMO: O processo de formação do profissional de saúde tem se modificado na busca de educar profissionais com uma visão integral e que proponham mudanças na sociedade. Dentro desse contexto, a saúde da pessoa com deficiência merece destaque, tendo em vista que essas pessoas têm mais barreiras de acessos aos serviços prestados por esses profissionais, tais como dificuldade de comunicaçáo, empatia do profissional, barreiras arquitetônicas, instrumentais, entre outras. Logo, este estudo objetiva analisar como duas universidades públicas discutem a saúde da pessoa com deficiência dentro do currículo do profissional de saúde. Para isso, analisaram-se projetos pedagógicos de oito cursos de Saúde. Concluiu-se que os projetos analisados não garantem uma formação integral no que tange à atenção à saúde da pessoa com deficiência.
\end{abstract}

PALAVRAS-CHAVE: Formação Profissional. Profissional da Saúde. Pessoa com deficiência. Ensino Superior.

\begin{abstract}
The process of training the health professional has changed in the search to educate professionals with an integral vision and who propose changes in the society. Within this context, the health of people with disabilities deserves to be highlighted, given that these people have more access barriers to the services provided by these professionals, such as communication difficulties, professional empathy, architectural, instrumental barriers, among others. Therefore, this study aims to analyze how two public universities discuss the health of the person with disability within the curriculum of the health professional. For this, we analyzed pedagogical projects of eight health courses. We concluded that the projects analyzed do not guarantee an integral training regarding the health care of the person with disability.
\end{abstract}

KEYWORDS: Professional qualification. Healthcare professional. Person with disability. Higher Education.

\section{INTRODUÇÁO}

Há uma discussão emergente, no Brasil, sobre o processo de educação dos profissionais de saúde na tentativa de formar esses profissionais com uma visão holística, humanista e

\footnotetext{
${ }^{1}$ https://doi.org/10.1590/1980-54702020v26e0181

${ }^{2}$ Mestre em Ciências pelo Programa de Pós-Graduação Ciências da Saúde e Biológicas da Universidade Federal do Vale do São Francisco. Petrolina/Pernambuco/Brasil. E-mail: marhlalba@gmail.com. ORCID: https://orcid.org/0000-0001-7996-6702

${ }^{3}$ Docente do colegiado de Educação Física, Universidade Federal Rural do Rio de Janeiro. Rio de Janeiro/Brasil. E-mail: francisnatally@yahoo.com.br. ORCID: https://orcid.org/0000-0001-5309-1186

${ }^{4}$ Docente permanente do Programa de Pós-Graduação em Psicologia, Universidade Federal do Vale do São Francisco. Petrolina/ Pernambuco/Brasil. E-mail: ramon.missias@univasf.edu.br. ORCID: https://orcid.org/0000-0003-1035-995X

${ }^{5}$ Discente do curso de Medicina na Universidade Federal do Vale do São Francisco. Petrolina/Pernambuco/Brasil. E-mail: albertinojfneto@gmail.com. ORCID: https://orcid.org/0000-0003-0237-8501

${ }^{6}$ Docente permanente do Programa de Pós-Graduação em Ciências da Saúde e Biológicas, Universidade Federal do Vale do São Francisco. Petrolina/Pernambuco/Brasil. E-mail: cheila.bedor@univasf.edu.br. ORCID: https://orcid.org/0000-0002-1614-7539
} 
crítica, que, pautados por princípios éticos, sejam capazes de refletir sobre a realidade econômica, política, social e cultural das comunidades. Além disso, é preciso que tenham como objetivo de atuação profissional contribuir para a melhoria da qualidade de vida e também da saúde dos indivíduos e das coletividades (Jurdi et al., 2017; Moreira \& Araújo Dias, 2015).

A necessidade de mudança no currículo nos cursos de Saúde tornou-se ainda mais evidente após o Movimento de Reforma Sanitária, iniciada na década de 1970, culminando na 8a Conferência Nacional de Saúde, em 1986. Na ocasião, o tema que emergiu foi "saúde é direito de todos e dever do Estado" (Ministério da Saúde, 2003), e, como consequência, em 1988, a nova Constituição Brasileira criou o Sistema Único de Saúde (SUS), regulamentado pela Lei Orgânica de Saúde (LOS) no 8.080, de 19 de setembro de 1990.

Com o advento do SUS, foi identificada a necessidade de implementar novas formas de cuidar, emergindo, desse contexto, uma reorganização dos sistemas de saúde e uma consequente diferenciação no processo de formação profissional, reforçada pelo princípio da integralidade. Logo, os recursos humanos em saúde são voltados à integração entre ensino-serviço (Lei no 8.080/1990). Em consonância com essa necessidade, foi elaborada a Lei de Diretrizes e Bases da Educação (Lei no 9.394, de 20 de dezembro de 1996), que trouxe, como característica, uma preocupação com a promoção de formação do profissional adequada à realidade e aos anseios da sociedade.

Em 2001, surgiram as Diretrizes Curriculares Nacionais (Resolução no 5, de 15 de março de 2011), como uma forma de tornar os currículos mais flexíveis e possibilitar às universidades diversas adaptaçóes dentro das suas necessidades e das transformaçóes sociais. Ainda no mesmo ano, foi aprovado o Parecer CNE/CES n ${ }^{\circ} 1.133 / 2001$, que traz como objeto das Diretrizes Curriculares Nacionais dos Cursos de Graduação em Enfermagem, Medicina e Nutriçáo, que permite

que currículos propostos possam construir perfil acadêmico e profissional com competências, habilidades e conteúdos, dentro de perspectivas e abordagens contemporâneas de formação pertinentes e compatíveis com referências nacionais e internacionais, capazes de atuar com qualidade, eficiência e resolutividade, no Sistema Único de Saúde (SUS), considerando o processo da Reforma Sanitária Brasileira. (Parecer CNE/CES no 1.133/2001, p. 4).

Percebe-se, então, que existe um esforço expresso para uma mudança curricular. Para tanto, é preciso a integração do sistema educacional, nesse caso, as universidades, com os sistemas e os serviços de saúde, com vistas a atender às necessidades da sociedade contemporânea. Desse modo, os Projetos Pedagógicos de Curso (PPCs) representam uma ferramenta de investigação de grande relevância nesse processo, uma vez que permitem a abordagem de questôes complexas relativas à formação profissional. Os projetos pedagógicos vão além do âmbito profissional, chegando a atingir as relações humanas, acolhimento e a qualidade de vida dos trabalhadores (Ceccim \& Ferla, 2008).

Assim sendo, os projetos de curso falam de si e de seus contextos, eles possuem posicionamentos teóricos para sustentar estratégias metodológicas, ao passo que criam processos com a finalidade de efetivar seus objetivos, que não são isolados, já que seguem políticas formativas bem definidas com o objetivo de potencializar as açóes do SUS. Ainda se posicio- 
nam teoricamente para sustentar suas estratégias metodológicas, criam e gerenciam processos buscando a efetivação de seus objetivos que, no caso deste estudo, não são singulares, já que os programas se inscrevem no viés de políticas formativas bem definidas em saúde, que têm, como objetivo, potencializar as açóes do SUS e formar profissionais na perspectiva de uma saúde integral (Gomes, 2016).

Nesse contexto, observa-se que a formação do profissional de saúde deve ser pautada nas necessidades da população. Mesmo com essa garantia de direitos, alguns estudos recentes vêm demonstrando uma ineficiência do atendimento dos profissionais de saúde às pessoas com deficiência (Baraúna \& Sales, 2018; Gotado \& Almeida, 2016; Martins, Costa, Rezende, Gomes, Dantas, \& Santos, 2015; Sousa \& Almeida, 2017; Resende, Nóbrega, \& Moreira, 2014). Nesse sentido, uma das causas apontadas está relacionada a uma formação inicial inadequada desses profissionais (Washington Institute of Medicine, 2007).

Recentemente, a Lei Brasileira de Inclusão (LBI) (Lei no 13.146, de 6 de julho de 2015) reafirma que é dever do Estado assegurar, à pessoa com deficiência, uma atenção integral, em todos os níveis de complexidade, por intermédio do SUS, com garantia de acesso universal e igualitário.

Considerando a importância temática discutida, bem como a escassez de literatura que realize a avaliação dos cursos de Saúde para integralidade do atendimento à pessoa com deficiência PcD, este artigo tem como objetivo analisar os PPCs dos cursos de formação inicial em saúde de duas universidades públicas da região do Submédio do São Francisco, no estado de Pernambuco, Brasil, no âmbito da atenção à saúde da pessoa com deficiência.

\section{Método}

Foi realizada pesquisa descritiva e exploratória, de caráter documental, na qual foram analisados os PPCs da área de saúde de duas universidades públicas do município de Petrolina. A escolha pelo referido cenário deu-se em virtude de estas serem as únicas universidades públicas a ofertarem cursos na área de saúde na referida região.

Para coleta dos dados, foram utilizados como instrumentos de pesquisa os PPCs dos cursos de Enfermagem, Medicina, Psicologia, Farmácia, Educação Física, Fisioterapia e Nutrição das duas universidades, identificadas como U1 e U2. Os PPCs foram lidos na íntegra e minuciosamente apreciados. Entretanto, como forma de sistematizar a análise, foi construído um instrumento com cinco blocos de análise, a saber: (1) Descrição geral do curso; (2) Disciplinas ofertadas; (3) Habilidades profissionais; (4) Competências profissionais; e (5) Discussão voltada à atenção à saúde da pessoa com deficiência. A coleta e a análise dos dados ocorreram entre junho de 2017 e fevereiro de 2018.

As informações oriundas na análise documental foram assistidas pela Técnica de Análise de Conteúdo (Bardin, 2011), e, para observância do procedimento de análise, utilizaram-se, como referenciais norteadores, as Diretrizes Curriculares Nacionais (Parecer CNE/CES $\left.\mathrm{n}^{\circ} 1.133 / 2001\right)$; e, no que tange às políticas relativas à saúde da pessoa com deficiência, a Lei $\mathrm{n}^{\circ}$ 13.146/2015, o Decreto no 7.612, de 17 de novembro de 2011; Portaria no 793, de 24 de abril de 2012; Portaria no 1.060 , de 5 de junho de 2002. A pesquisa seguiu todos os preceitos 
éticos e legais e, nesse sentido, foi aprovada pelo Comitê de Ética em Pesquisa (CEP) sob o Parecer $n^{\circ} 2.339 .386$.

\section{Resultados E discussáo}

Após a leitura e a análise temática dos currículos dos cursos em voga, emergiram três temáticas: (1) Os Cursos de Saúde no Vale do São Francisco; (2) Habilidades e competências dos futuros profissionais de Saúde; (3) A deficiência e a formação dos profissionais de Saúde.

\subsection{Os cursos de Saúde no Vale do Sáo Francisco}

Nessa temática, foram elencados, por meio dos PPCs, o ano de criação e de reformulação curricular, a carga horária total, perfil dos egressos e objetivos. Foram identificados oito cursos de Saúde, sendo cinco em uma universidade identificada como U1 e três em U2. O único curso em comum nas duas universidades é o de Enfermagem, que será identificado como E1 e E2. Os PPCs foram escritos entre 2008 e 2013, sendo o documento do curso de Medicina o que tem a reformulação mais antiga (2008), e o plano de E1, o mais recente (2013), como pode ser observado no Quadro 1.

\begin{tabular}{|l|c|c|c|l|}
\hline \multicolumn{1}{|c|}{ Cursos } & Universidade & $\begin{array}{c}\text { Ano de criaçáo } \\
\text { do curso }\end{array}$ & $\begin{array}{c}\text { Ano do PPC } \\
\text { analisado }\end{array}$ & \multicolumn{1}{|c|}{$\begin{array}{c}\text { Disponibilidade do } \\
\text { PPC }\end{array}$} \\
\hline Enfermagem U1 & $\mathrm{U} 1$ & 2002 & 2013 & Sítio eletrônico \\
\hline Medicina & $\mathrm{U} 1$ & 2004 & 2008 & Sítio eletrônico \\
\hline Psicologia & $\mathrm{U} 1$ & 2005 & 2010 & Sítio eletrônico \\
\hline Farmácia & $\mathrm{U} 1$ & 2008 & 2012 & Secretaria do curso \\
\hline Educação Física & $\mathrm{U} 1$ & 2008 & 2012 & Secretaria do curso \\
\hline Fisioterapia & $\mathrm{U} 2$ & 2006 & 2010 & Sítio eletrônico \\
\hline Enfermagem U2 & $\mathrm{U} 2$ & 2002 & 2011 & Sítio eletrônico \\
\hline Nutrição & $\mathrm{U} 2$ & 2010 & 2013 & Sítio eletrônico \\
\hline
\end{tabular}

Quadro 1: Caracterização quanto à criação do PPC dos cursos de Saúde em duas universidades públicas no Submédio do São Francisco (2018)

Fonte: Elaborado pelas autoras

As primeiras DCN para os cursos de Saúde foram lançadas entre os anos de 2001 e 2004. Entretanto, os cursos de Medicina e Farmácia realizaram a atualização dos PPCs em 2008 e 2012, respectivamente, e não referenciaram as novas DCN, que são de 2014 e 2017 . O fato reforça, pois, a necessidade de avaliação periódica e permanente dos PPCs, como estratégia de superação para as lacunas identificadas. 
Quanto aos objetivos dos cursos, as universidades descrevem a formação ética e generalista, com o enfoque na visão holística para com os indivíduos que necessitarem de seus cuidados, como descritos a seguir:

Assegurar ao graduando em Ciências Farmacêuticas, uma formação generalista, capacitando-o a atuar em todos os níveis de atenção à saúde, com uma visão ampla e global, respeitando os princípios éticos e bioéticos, morais, religiosos e socioculturais do indivíduo e da coletividade, com o objetivo de prevenir, preservar e promover a saúde individual e coletiva. (PPC de Farmácia, 2012).

Formar profissionais enfermeiros(as) aptos a cuidar do ser humano, família, grupos e comunidade em situaçóes de saúde e doença, no âmbito da gestão, gerência, supervisão e avaliação no Sistema Único de Saúde (PPC de Enfermagem E2, 2011).

Formação de um profissional de saúde apto ao trabalho em uma equipe interdisciplinar, com ênfase na integralidade no cuidado dos pacientes (PPC de Fisioterapia, 2010).

Formar enfermeira(os) cidadãos que utilizem a ética e o conhecimento técnico científico para atuar nas diversas áreas do conhecimento e prestar cuidado integral à saúde do indivíduo, da família e da comunidade (PPC de Enfermagem E1, 2013).

Formar profissionais aptos a analisar criticamente a realidade social em que vivem; assegurar uma formação generalista, humanista e crítica, qualificadora da intervenção acadêmico-profissional, fundamentada no rigor científico, na reflexão filosófica e na conduta ética (PPC de Educação Física, 2012).

Formação de um profissional ético e competente tecnicamente, sem prejuízo humanístico e apto, para participar efetivamente das transformaçôes sociais desta região (PPC de Medicina, 2008).

Formar nutricionistas generalistas, capazes de aplicar a ciência da Alimentação e Nutrição para a melhoria dos níveis de saúde e qualidade de vida da população da regiáo e brasileira (PPC de Nutrição, 2013).

Formar profissionais críticos, reflexivos e socialmente comprometidos, capazes de construir e contextualizar saberes e práticas, promovendo e divulgando o conhecimento de forma sistematizada para as comunidades (PPC de Psicologia, 2010).

Durante a etapa de análise documental, percebeu-se o empenho das universidades em descreverem a importância da formação de um profissional ético, comprometido com a integralidade da saúde dos indivíduos e da coletividade. Com isso, é possível afirmar que os PPCs dos cursos estudados se encontram em conformidade com as DCN e, nesse sentido, refletem a real necessidade da população para bom atendimento. Esse comprometimento também foi descrito em um estudo realizado em uma Universidade do Centro-Oeste, no qual foi observado que todos os PPCs analisados atendiam às premissas gerais das DCN e das políticas indutoras de formação em saúde (Moraes \& Costa, 2016).

Contudo, mesmo com o PPC construído com a preocupação de atender às DCN, há, ainda, grande obstáculo a ser enfrentado: a sua implementação na prática. As DCN propostas para a formação do profissional de Saúde têm alcançado as metas e encontra-se no caminho da construção de uma cultura de mudança significativa. Entretanto, é necessário ter consciência de que é preciso superar este obstáculo supramencionado (Keller-Franco, Kuntze, \& Costa, 2012), a fim de que sejam estimuladas as açóes e as práticas mais eficientes no processo de formulação didática dos PPCs dos cursos em análise. 
Um outro aspecto que emergiu durante a análise desses documentos foram as habilidades e as competências que os cursos de Saúde trazem em seus PPCs, as quais serão descritas na próxima temática.

\subsection{Habilidddes E COMPETÊNCIAS NECESSÁRIAS À FORMAÇÃO PROFISSIONAL PARA UM ATENDIMENTO EFICAZ À PESSOA COM DEFICIÊNCIA}

Os atendimentos em saúde às pessoas com deficiência são apontados como falhos, tanto pela comunicação ineficaz (Batista, 2012), quanto pela inacessibilidade arquitetônica (Martins et al., 2015), assim como por presunçóes e percepçóes sobre a deficiência, podendo esta, inclusive, provocar ameaças à saúde dessa pessoa (Shakespeare, Iezzoni, \& Groce, 2009).

Uma das possibilidades de minimizar essas barreiras de acesso aos serviços de saúde seriam as organizaçóes educacionais, formadoras de futuros profissionais, incluírem esses conteúdos temáticos em seus currículos e que os serviços de saúde estimulassem a capacitação de seus profissionais para assim adquirirem habilidades comunicativas. Ademais, deveriam adequar a estrutura física, de modo que as necessidades desses indivíduos, no ambiente hospitalar, fossem atendidas de forma integral e humanizada (Martins et al., 2015).

Conforme percebido, a minimização dessas barreiras demanda uma formação inclusiva e integral para que o futuro profissional de saúde possa desenvolver a aptidão no atendimento de todos os indivíduos que necessitarem de seus cuidados. Entretanto, o que se encontra perceptível na formação dos profissionais, em geral, é que existe um espaço vazio, especialmente no que diz respeito à formação no âmbito da atenção à saúde da pessoa com deficiência (Costa, 2015). Existe, então, uma dicotomia entre o que se espera diante da atenção integral e a efetiva formação dos profissionais de saúde. Por essa razão, torna-se necessário entender que as competências e as habilidades estão sendo descritas pelas universidades e de que forma as instituições de ensino influenciam na atenção integral e universal dos sujeitos com deficiência.

São descritas, no Quadro 2, as habilidades e as competências gerais que os profissionais de saúde devem ter, de acordo com os PPCs analisados.

\begin{tabular}{|c|c|c|c|c|c|c|c|c|}
\hline \multicolumn{9}{|c|}{ CuRSOS DE SAÚdE } \\
\hline $\begin{array}{l}\text { Habilidades e } \\
\text { competências }\end{array}$ & $\begin{array}{c}\text { Enferma- } \\
\text { gem } 1\end{array}$ & Medicina & Psicologia & Farmácia & $\begin{array}{c}\text { Educaçáo } \\
\text { Física }\end{array}$ & $\begin{array}{c}\text { Fisiote- } \\
\text { rapia }\end{array}$ & $\begin{array}{c}\text { Enferma- } \\
\text { gem } 2\end{array}$ & Nutrição \\
\hline Atençấo à saúde & $\mathrm{x}$ & $\mathrm{x}$ & & $\mathrm{x}$ & $\mathrm{x}$ & $\mathrm{x}$ & $\mathrm{x}$ & $\mathrm{x}$ \\
\hline Tomada de decisão & $\mathrm{x}$ & $\mathrm{x}$ & $\mathrm{x}$ & $\mathrm{x}$ & $\mathrm{x}$ & $\mathrm{x}$ & $\mathrm{x}$ & $\mathrm{x}$ \\
\hline Comunicação & $\mathrm{x}$ & $\mathrm{x}$ & $\mathrm{x}$ & $\mathrm{x}$ & $\mathrm{x}$ & $\mathrm{x}$ & $\mathrm{x}$ & $\mathrm{x}$ \\
\hline Liderança & $\mathrm{x}$ & $\mathrm{x}$ & & $\mathrm{x}$ & $\mathrm{x}$ & $\mathrm{x}$ & $\mathrm{x}$ & $\mathrm{x}$ \\
\hline $\begin{array}{l}\text { Administração e } \\
\text { gerenciamento }\end{array}$ & $\mathrm{x}$ & $\mathrm{x}$ & & $\mathrm{x}$ & $\mathrm{x}$ & $\mathrm{x}$ & $\mathrm{x}$ & $\mathrm{x}$ \\
\hline $\begin{array}{l}\text { Educação perma- } \\
\text { nente }\end{array}$ & $\mathrm{x}$ & $\mathrm{x}$ & $\mathrm{x}$ & $\mathrm{x}$ & & $\mathrm{x}$ & $\mathrm{x}$ & $\mathrm{x}$ \\
\hline $\begin{array}{l}\text { Interação com a } \\
\text { sociedade }\end{array}$ & & & & & $\mathrm{x}$ & $\mathrm{x}$ & & \\
\hline $\begin{array}{l}\text { Conhecer as neces- } \\
\text { sidades e a realidade } \\
\text { social }\end{array}$ & $\mathrm{x}$ & & & $\mathrm{x}$ & $\mathrm{x}$ & & & \\
\hline
\end{tabular}




\begin{tabular}{|l|c|c|c|c|c|c|c|c|}
\hline $\begin{array}{l}\text { Garantir a integrali- } \\
\text { dade da assistência }\end{array}$ & $\mathrm{x}$ & $\mathrm{x}$ & $\mathrm{x}$ & $\mathrm{x}$ & & $\mathrm{x}$ & & $\mathrm{x}$ \\
\hline $\begin{array}{l}\text { Atuação multidisci- } \\
\text { plinar }\end{array}$ & $\mathrm{x}$ & $\mathrm{x}$ & $\mathrm{x}$ & $\mathrm{x}$ & $\mathrm{x}$ & $\mathrm{x}$ & $\mathrm{x}$ & $\mathrm{x}$ \\
\hline
\end{tabular}

Quadro 2: Habilidades e competências gerais dos cursos de saúde analisados Fonte: Elaborado pelas autoras

Diante das competências e das habilidades apresentadas, percebe-se que a Tomada de decisão, Comunicação e Atuação multiprofissional foram as competências comuns a todos os profissionais de saúde.

Um estudo piloto realizado por Castro, Rowe, Andrade e Cyrino (2017) mostrou que as competências generalistas, como as encontradas nesta pesquisa, também podem ser utilizadas para a formação de profissionais de saúde que atendam às necessidades de saúde da pessoa com deficiência. Kirshner e Curry (2009) foram mais específicos e propuseram seis competências essenciais para a formaçáo relacionada à deficiência: (1) Enquadrar a deficiência no contexto da diversidade humana ao longo da vida, dentro do social e em ambientes culturais; (2) Treinamento para avaliação da deficiência e a real consequência das condiçóes de saúde; (3) Formação para cuidados comportamentais na interação com a pessoa com deficiência; (4) Aprender sobre outros profissionais de saúde, para formar equipes integradas; (5) Compreensão dos aspectos legais juntamente aos princípios de desenho universal; e (6) Abordagem de cuidados centradas no cliente, incluindo a percepção destes sobre qualidade de vida. Algumas dessas competências corroboram as encontradas nesta pesquisa. Nesse sentido, espera-se que os profissionais tenham competências e habilidades suficientes para atender às pessoas com deficiência.

Para reforçar essa formação, com vistas à saúde da pessoa com deficiência, também foram avaliadas as disciplinas e as discussóes sobre a sua saúde nos PPCs, cujos resultados são apresentados na próxima categoria.

\subsection{Oferta de disciplinas e discussóes relacionadas à saúde da pessoa com DEFICIÊNCIA NOS PPCs dos CURSOS DE SAÚdE}

Diante do processo de formação dos profissionais de saúde apoiado na literatura, é importante analisar como esses profissionais estâo sendo formados para a atenção à saúde da pessoa com deficiência. A partir dessa percepção, buscou-se identificar quais os cursos que ofereciam Libras como disciplina optativa ou obrigatória; se estes mencionavam, em suas referências bibliográficas, a Política Nacional de Saúde da Pessoa Portadora de Deficiência (2008, 2010) em suas duas versóes, além de disciplinas que descrevessem, na Ementa, o conteúdo relacionado à atenção da pessoa com deficiência. Os resultados estáo apresentados no Quadro 3 que segue. 


\begin{tabular}{|l|c|c|}
\hline \multicolumn{1}{|c|}{ Cursos } & Oferta de Libras & Referência à Política de Saúde \\
\hline Enfermagem (U1) & Não & Não \\
\hline Enfermagem (U2) & Não & Não \\
\hline Medicina & Não \\
\hline Psicologia & Não & Não \\
\hline Farmácia & Sim & Não \\
\hline Fisioterapia & Não & Não \\
\hline Nutrição & Sim & Não \\
\hline Educação física & Sim & Não \\
\hline
\end{tabular}

Quadro 3: Análise dos PPC dos cursos estudados quanto à oferta de Libras e referência à Política Nacional da Pessoa com Deficiência nos cursos de Saúde do submédio do São Francisco Fonte: Elaborado pelas autoras

Tratando-se do ensino de Libras nos cursos de Saúde pesquisados, 62,5\% dos PPCs não apresentam Libras como disciplina optativa, e nenhum deles apresenta como disciplina obrigatória. Esse resultado contraria o Decreto no 5.626, de 22 de dezembro de 2005, que estabelece que, nos cursos de formação de professores para o exercício do magistério, em nível médio e superior, e, nos cursos de Fonoaudiologia, a linguagem de sinais deve ser inserida como uma disciplina obrigatória e, nos demais cursos de Educação Superior e Profissional, deve ser disciplina optativa.

Existe divergência quando se observa o ensino de Libras e a proposta das DCN, em que uma das competências e habilidades que deve ser garantida na formação do profissional de saúde é a comunicação. Todos os cursos de Saúde analisados, exceto Educação Física, trazem, nas DCN, o seguinte texto:

Comunicação: os profissionais de saúde devem ser acessíveis e devem manter a confidencialidade das informaçôes a eles confiadas, na interação com outros profissionais de saúde e o público em geral. A comunicaçáo envolve comunicação verbal, não verbal e habilidades de escrita e leitura; o domínio de, pelo menos, uma língua estrangeira e de tecnologias de comunicação e informação (Parecer CNE/CES no 1.133, 2001; Resolução CNE/CNS no 3, 2001; Resolução CNE/ CES no 5, 2001; Resolução CNE/CES 4, 2002; Resolução no 5, 2011; Resolução no 3, 2014; Resolução no 6, 2017).

Esse excerto mostra a importância da comunicação no processo formativo desses profissionais e intensifica a necessidade de atendimento, não somente às DCN, mas também ao processo de integralidade e de humanização do cuidado, já que o processo comunicativo é um dos principais desafios para humanização da saúde (Deslandes \& Mitre, 2009). Sobre isso, Dias, Coutinho, Gaspar, Moeller e Mamede (2017), em estudo, explicitaram que a dificuldade de estabelecimento da relação médico-paciente gera frustrações e também infrequências dos pacientes aos serviços de assistência à saúde. 
É constatado que as instituições públicas de Ensino Superior não estão ofertando oportunidades do ensino da Libras para os seus alunos nos cursos de saúde. Isso resulta em um processo de comunicação falho e consequente danos à saúde das pessoas surdas. Logo, este artigo reforça a importância do ensino de Libras como disciplina obrigatória e não como optativa, nos currículos dos profissionais de saúde, corroborando estudos anteriores (Ramos \& Almeida, 2017; Souza \& Porrozzi, 2017).

Quanto ao ensino da Política Nacional de Atenção à Saúde da Pessoa com Deficiência, fica evidente a não preocupação com a discussão dessa temática nos cursos de saúde, uma vez que nenhum curso das Instituiçóes pesquisadas a colocou nas referências a serem trabalhadas com os futuros profissionais de saúde. Isso leva ao desconhecimento por parte destes sobre as necessidades específicas desse grupo populacional.

Das Universidades pesquisadas, foram encontradas apenas duas disciplinas entre todos os cursos de saúde, conforme mostra o Quadro 4.

\begin{tabular}{|c|c|c|c|c|}
\hline Curso & Disciplina & $\begin{array}{l}\text { Carga } \\
\text { horária } \\
\text { do curso } \\
\end{array}$ & $\begin{array}{l}\text { Carga horária } \\
\text { da disciplina }\end{array}$ & Modalidade \\
\hline \multirow{2}{*}{ Medicina } & Introdução à Medicina & \multirow{2}{*}{7320 horas } & 30 horas & Obrigatória \\
\hline & Medicina geral e da criança I & & 60 horas & Obrigatória \\
\hline \multirow{2}{*}{ Educação Física } & $\begin{array}{l}\text { Educação Física para portadores de } \\
\text { necessidades especiais }\end{array}$ & \multirow{2}{*}{3200 horas } & 60 horas & Obrigatória \\
\hline & Libras & & 60 horas & Optativa \\
\hline \multirow{3}{*}{ Psicologia } & Psicologia e pessoa com deficiência & \multirow{3}{*}{4045 horas } & 60 horas & Optativa \\
\hline & Libras & & 60 horas & Optativa \\
\hline & Práticas Integrativas & & 60 horas & Obrigatória \\
\hline Farmácia & Psicologia e Relaçóes humanas & 4800 horas & 30 horas & Obrigatória \\
\hline Nutriçáo & Libras básica & 3510 horas & 30 horas & Eletiva \\
\hline Enfermagem (U1) & Não há nenhuma disciplina & 4530 horas & - & - \\
\hline Enfermagem (U2) & Não há nenhuma disciplina & 4100 horas & - & - \\
\hline Fisioterapia & Não há nenhuma disciplina & 4000 horas & - & - \\
\hline
\end{tabular}

Quadro 4: Disciplinas específicas que trabalham com o tema e que descrevem nas ementas a atenção à saúde da pessoa com deficiência nos cursos de Saúde do submédio do São Francisco Fonte: Elaborado pelas autoras

Foge de o escopo desta pesquisa identificar disciplinas que diagnostiquem a deficiência ou a remetam como patologia. O objetivo aqui apresentado é a discussão de políticas e açóes que melhorem o acesso desses indivíduos aos serviços de saúde, que teçam discussóes, com o intuito de favorecer à humanização do atendimento, na perspectiva social da deficiência. Dos oito PPCs analisados, apenas 25\% traziam cada um uma disciplina específica que discute 
a atenção à saúde da pessoa com deficiência, são elas: Psicologia e pessoas com deficiência e Educação Física para portadores de necessidades especiais. As demais, foram identificadas por trazerem dentro da ementa conteúdos sobre a atenção à saúde da pessoa com deficiência a serem abordados durante a disciplina.

Quando avaliada a carga horária dessas disciplinas, destaca-se que os cursos de saúde das Universidades estudadas reservam apenas 1,1\% de sua carga horária, variando de 30 a 60 horas, para discussão acerca da temática da saúde da pessoa com deficiência. Um estudo realizado com escolas médicas da Austrália (Trollor et al., 2016) descreve que essas faculdades reservam cerca de 2 horas e 55 minutos de conteúdo sobre deficiência intelectual. $\mathrm{O}$ artigo enfatiza, ainda, que apenas um pequeno número de Universidades oferta essa temática em seus conteúdos obrigatórios (Trollor et al., 2016).

Essa inserção de disciplinas/conteúdos já é discutida desde a Portaria ${ }^{\circ} 1.793$, de dezembro de 1994, que recomenda a inclusão de conteúdos relativos aos aspectos "Ético-políticoeducacionais da normalização e integração da pessoa portadora de necessidades especiais" nos cursos do grupo de Ciência da Saúde.

Uma possibilidade para discussão sobre a atenção à saúde da pessoa com deficiência nos cursos de saúde seria envolver o contexto de forma transversal e/ou inclusão de disciplinas que tenham foco na atenção à saúde da pessoa com deficiência. Entretanto, essas possibilidades também não são absorvidas pelas Universidades estudadas tendo em vista que somente um curso de enfermagem relata que o tema será trabalhado transversalmente. Entretanto, no próprio texto do PCC, já é descrita a falha da referida transversalidade: "Admite-se a necessidade de aprofundar nessas discussóes para que mais iniciativas sejam adotadas nos módulos no intuito de integrar cada vez mais a nuances da atenção à saúde da pessoa com deficiência” (PPC de Enfermagem, 2013, p. 128).

Esse tipo de discussão transversal é o desejado que aconteça em todos os cursos; no entanto, é necessário estudo mais aprofundado para saber de que forma a temática está sendo inserida e discutida, uma vez que, na análise do PPC, não foi encontrado descrito o conteúdo nem na ementa, nem em suas referências, o que deixa uma lacuna sobre o que está sendo discutido de fato no curso supracitado. É preciso ressaltar que, para o melhor atendimento em saúde às pessoas com deficiência por profissionais da área, necessita-se ir além da inserção esporádica de disciplinas, pois a temática deve ser discutida e trabalhada no cotidiano dos profissionais em formação.

Um exemplo disso foi o estudo de Symons, Morley, McGuigan e Akl (2014) que comparou a formação de dois grupos de estudantes de Medicina: um grupo, cujo curso utilizava um currículo longitudinal para cuidar de pessoa com deficiência; e outro sem essa especificidade. Os resultados apontam que o currículo longitudinal levou a uma melhora significativa de vários fatores relacionados ao conforto e à atitude dos estudantes envolvidos na pesquisa, futuros profissionais, em relação a essas pessoas (Symons et al., 2014).

A pesquisa de Sullivan e Mendonca (2017) comparou o contato dos estudantes de terapia ocupacional à pessoa com deficiência por meio de palestras ou em trabalho de campo. As autoras concluíram que o contato direto (o trabalho de campo) melhorou significativamente as atitudes dos estudantes em relação à pessoa com deficiência (Sullivan \& Mendonca, 2017). 
Desse modo, práticas pontuais, como palestras, podem não trazer o efeito desejado na mudança de paradigma e de atitudes dos profissionais de saúde em relação às pessoas com deficiência. Nesse sentido, atenta-se para a importância do contato com a pessoa com deficiência como disparador de modificação para o atendimento em saúde a esse público. Um desses contatos pode ser vivenciado no estágio curricular. Contudo, durante análise de projetos pedagógicos, nenhuma das universidades estudadas reserva carga horária específica dentro das propostas de estágio, tampouco descreve que esse atendimento será incluído aos estágios já estabelecidos.

No atendimento, o contato prévio provoca mudanças de atitudes. A vivência do estágio pode revelar a falta de familiaridade com a realidade da pessoa com deficiência e o encontro com as diferenças (Araujo, Santos, Rosa, \& Gomes, 2014). O estágio pode reduzir os aspectos negativos, de moda a permitir a redução de concepçóes prévias que dão origem ao estigma e à quebra de uma visão social única e viabilizar que o aluno veja as possibilidades do indivíduo e não as desgraças. Traz, além de valores pessoais para formação humana, recursos profissionais incomparáveis, com vistas à integralidade (Araujo et al., 2014).

Nesse contexto, é inquestionável a necessidade de repensar-se a formação dos profissionais de saúde. A necessidade foi também evidenciada no primeiro Relatório Mundial sobre Deficiência de 2011 (World Health Organization [WHO], 2011), que deixou explícito que os profissionais de saúde necessitam de conhecimentos e habilidades adequadas sobre as condiçóes de comorbidades associadas à deficiência do sujeito, bem como de gerir as necessidades de cuidado em saúde das pessoas com deficiência. Esta tem foco em dois objetivos: atender às necessidades de saúde da pessoa com deficiência e compreender que o atendimento adequado a essas pessoas é um direito humano. Além disso, as atitudes discriminatórias e equívocos implícitos são açóes que precisam ser desafiadas (Shakespeare \& Kleine, 2013).

Ainda assim, é evidente que as universidades públicas estudadas, mesmo com um PPC enfatizando o compromisso com a formação de um profissional comprometido com a integralidade da saúde dos indivíduos e da coletividade, não discute a atenção à saúde da pessoa com deficiência e negligencia o cuidado à essa enorme parcela da população. Portanto, espera-se que haja uma atualização, não só dos PPCs, com vistas a cumprir leis e decretos, mas que haja mudança de pensamentos e de atitudes da comunidade acadêmica, que a discussão seja contínua para a formação, de fato, de profissionais de saúde preparados para atenção à saúde de toda população.

\section{Conclusão}

Os cursos de Saúde estudados não possuem componentes curriculares específicos em seus PPCs que garantam uma formação integral e universal quando se trata de atenção à saúde da pessoa com deficiência. Nesse sentido, é necessário que haja reformulaçáo dos PPCs em busca de interface desse conhecimento e que os profissionais de saúde conheçam as demandas de saúde da pessoa com deficiência, formas de comunicação e que esse conhecimento quebre o estigma, de modo a gerar atitudes positivas e, consequentemente, melhorar a saúde dessa população.

Este estudo aponta que o ensino sobre deficiência não deve se limitar à oferta de disciplinas isoladas; é necessário de fato que seja algo longitudinal, ou seja, ao logo do curso, 
porém que essa obrigatoriedade esteja expressa no PPC. Percebe-se também que o ensino de Libras deve ser um componente obrigatório aos profissionais de saúde, tendo em vista a importância da comunicação no processo de saúde e na atuação desses profissionais.

Esta pesquisa possibilitará que as Universidades não só realizem análise dos currículos de saúde em uso, como também promovam uma reflexão nas mudanças, possibilitando um atendimento integral e favorecendo a atenção à saúde da pessoa com deficiência. Uma das limitaçóes desse estudo foi não avaliar o impacto e as atitudes que esses estudantes têm em relação às pessoas com deficiência, bem como não avaliar demais atividades de pesquisa e extensão que possam estar sendo desenvolvidas nas universidades. Tendo em vista o reduzido número de estudos realizados nos Brasil sobre a temática descrita, é necessário que mais análises sejam realizadas e que essas pesquisas sejam acompanhadas de mudanças curriculares para a efetiva formação de um profissional integral.

\section{REFERÊNCIAS}

Araujo, L. D. D., Santos, A. L. D. S. F., Rosa, A., \& Gomes, M. C. (2014). O estágio curricular como práxis pedagógica: representaçóes sociais acerca da criança com deficiência físico-motora entre estudantes de Fisioterapia. Interface-Comunicação, Saúde, Educação, 18, 151-164. DOI: https:// doi.org/10.1590/1807-57622014.0114

Baraúna, M. T., \& Sales, M. A. (2018). Assistência da pessoa com deficiência em uma clínica-escola. Plurais-Revista Multidisciplinar, 1(1), 74-87.

Bardin L. (2011). Análise de conteúdo. São Paulo: Ediçóes 70.

Batista, N. A. (2012). Educação interprofissional em saúde: concepções e práticas. Caderno Fnepas, 2(1), 25-28.

Castro, S. S., Rowe, M., Andrade, L. F., \& Cyrino, E. G. (2017). Developing competencies among health professions students related to the care of people with disabilities: a pilot study. InterfaceComunicação, Saúde, Educação, 22(65), 551-563. DOI: https://doi.org/10.1590/180757622016.0684

Ceccim, R. B., \& Ferla, A. A. (2008). Educação e saúde: ensino e cidadania como travessia de fronteiras. Trabalho, educaçâo e saúde, 6(3), 443-456. DOI: https://doi.org/10.1590/S198177462008000300003

Costa, L. S. M. (2015). Inclusão no curso médico: atenção integral à saúde das pessoas com deficiência. Rio de Janeiro: H. P. Comunicação.

Decreto $n^{\circ}$ 5.626, de 22 de dezembro de 2005. Regulamenta a Lei no 10.436, de 24 de abril de 2002, que dispóe sobre a Língua Brasileira de Sinais - Libras, e o art. 18 da Lei no 10.098, de 19 de dezembro de 2000. Recuperado em 3 de julho de 2017 de http://www.planalto.gov.br/ccivil_03/_Ato20042006/2005/Decreto/D5626.htm

Decreto $n^{\circ}$ 7.612, de 17 de novembro de 2011. Institui o Plano Nacional de direitos da pessoa com deficiência-Plano Viver Sem Limites. Recuperado em 3 de julho de 2017 de http://www.planalto. gov.br/ccivil_03/_Ato2011-2014/2011/Decreto/D7612.htm

Deslandes, S. F., \& Mitre, R. M. D. A. (2009). Processo comunicativo e humanização em saúde. Interface-Comunicação, Saúde, Educação, 13, 641-649. DOI: https://doi.org/10.1590/S141432832009000500015 
Dias, A. R., Coutinho, C. R., Gaspar, D. R., Moeller, L., \& Mamede, M. (2017). Libras na formação médica: possibilidade de quebra da barreira comunicativa e melhora na relação médico-paciente surdo. Revista de Medicina, 96(4), 209-214. DOI: http://dx.doi.org/10.11606/issn.1679-9836. v96i4p209-214

Gomes, M. Q. (2016). A construção de projetos pedagógicos na formação de profissionais da saúde. IJHE-Interdisciplinary Journal of Health Education, 1(1), 13-22. DOI: 10.4322/ijhe2016003

Gotado, R. G., \& Almeida, M. A. P. T. (2016). O acolhimento oferecido pelos Serviços de Saúde às pessoas com Necessidades Especiais. Id on line Revista Multidisciplinar e de Psicologia, 10(32), 126137. DOI: https://doi.org/10.14295/idonline.v7i19.218

Jurdi, A. P. S., Nicolau, S. M., Figueiredo, L. D. R. U., Rossit, R. A. S., Maximino, V. S., \& Borba, P. L. D. O. (2017). Revisitar processos: revisão da matriz curricular do curso de Terapia Ocupacional da Universidade Federal de São Paulo. Interface-Comunicação, Saúde, Educação, 22(65), 527-538. DOI: https://doi.org/10.1590/1807-57622016.0824

Keller-Franco, E., Kuntze, T. D., \& Costa, L. S. (2012). Inovação curricular na formação dos profissionais da saúde. Revista e-Curriculum, 8(2), 1-14.

Kirschner, K. L., \& Curry, R. H. (2009). Educating health care professionals to care for patients with disabilities. Jama, 302(12), 1334-1335. DOI: 10.1001/jama.2009.1398

Lei no 13.146, de 6 de julho de 2015. Institui a Lei Brasileira de Inclusão da Pessoa com Deficiência. Recuperado em 1 de julho de 2018 de http://www.planalto.gov.br/ccivil_03/_ato2015-2018/2015/ lei/l13146.htm

Lei $n^{\circ}$ 8.080, de 19 de setembro de 1990. Dispóe sobre as condiçóes para a promoçáo, proteção e recuperação da saúde, a organização e o funcionamento dos serviços correspondentes e dá outras providências. Recuperado em 1 de julho de 2018 de http://www.planalto.gov.br/ccivil_03/leis/ L8080.htm

Lei no 9.394, de 20 de dezembro de 1996. Estabelece as diretrizes e bases da educação nacional. Recuperado em 1 de julho de 2018 de http://www.planalto.gov.br/ccivil_03/leis/19394.htm

Martins, K. P., Costa, K. N. F. M., Rezende, L. C. M., Gomes, T. M., Dantas, T. R. A., \& Santos, S. R. (2015). Percepção da equipe de enfermagem acerca da acessibilidade física e de comunicação de pessoas com deficiência visual. Ciencia, Cuidado e Saúde, 14(2), 1019-1026. DOI: 10.4025/ cienccuidsaude.v14i2.22045

Ministério da Saúde. (2003). O desenvolvimento do Sistema Único de Saúde: avanços, desafios e reafirmação dos seus princípios e diretrizes. Brasília: MS.

Moraes, B. A., \& Costa, N. M. S. (2016). Compreendendo os currículos à luz dos norteadores da formação em saúde no Brasil. Revista da Escola de Enfermagem da USP, 50, 9-16. DOI: https:// doi.org/10.1590/S0080-623420160000300002

Moreira, C. O. F., \& de Araújo Dias, M. S. (2015). Diretrizes curriculares na saúde e as mudanças nos modelos de saúde e de educação. ABCS Health Sciences, 40(3), 300-305. DOI: https://doi. org/10.7322/abcshs.v40i3.811

Parecer CNE/CES n $n^{\circ}$ 1.133/2001. Diretrizes Curriculares Nacionais dos Cursos de Graduação em Enfermagem, Medicina e Nutrição. Recuperado em 1 de julho de 2018 de http://portal.mec.gov. br/dmdocuments/ces1133.pdf 
Portaria no 1.060, de 5 de junho de 2002. Política Nacional de Saúde da Pessoa Portadora de Deficiência. Recuperado em 1 de julho de 2018 de http://bvsms.saude.gov.br/bvs/saudelegis/gm/2002/ prt1060_05_06_2002.html

Portaria $n^{\circ} 1.793$, de dezembro de 1994. Recuperado em 1 de julho de 2018 de http://portal.mec.gov.br/ arquivos/pdf/portaria1793.pdf

Portaria $n^{\circ} 793$, de 24 de abril de 2012. Institui a rede de cuidados a pessoa com deficiência no âmbito do SUS. Recuperado em 1 de julho de 2018 de http://bvsms.saude.gov.br/bvs/saudelegis/gm/2012/ prt0793_24_04_2012.html

Ramos, T. S., \& Almeida, M. A. P. T. (2017). A Importância do ensino de Libras: Relevância para Profissionais de Saúde. Id on line Revista Multidisciplinar e de Psicologia, 10(33), 116-126. DOI: https://doi.org/10.14295/idonline.v10i33.606

Resende, A. C., da Nóbrega, S., \& Moreira, T. (2014). Experiência das pessoas com deficiência nos serviços de saúde: contribuição para a formação profissional. Artigo apresentado no Congresso Internacional de Humanidades \& Humanização em Saúde, São Paulo, Brasil. Recuperado em 4 de julho de 2018 de http://pdf.blucher.com.br.s3-sa-east-1.amazonaws.com/medicalproceedings/cihhs/10308.pdf

Resolução CNE/CES no 3, de 7 de novembro de 2001. Institui Diretrizes Curriculares Nacionais do Curso de Graduação em Enfermagem. Recuperado em 4 de julho de 2018 de http://portal.mec.gov.br/ cne/arquivos/pdf/CES03.pdf

Resolução no 6, de 19 de outubro de 2017. Institui as Diretrizes Curriculares Nacionais do Curso de Graduação em Farmácia e dá outras providências. Recuperado em 4 de julho de 2018 de http:// portal.mec.gov.br/docman/outubro-2017-pdf/74371-rces006-17-pdf/file

Resolução CNE/CES no 4, de 19 de fevereiro de 2002. Institui Diretrizes Curriculares Nacionais do Curso de Graduação em Fisioterapia. Recuperado em 4 de julho de 2018 de http://portal.mec.gov.br/cne/ arquivos/pdf/CES042002.pdf

Resoluçâo CNE/CES no 5, de 7 de novembro de 2001. Institui Diretrizes Curriculares Nacionais do Curso de Graduação em Nutrição. Recuperado em 4 de julho de 2018 http://portal.mec.gov.br/ cne/arquivos/pdf/CES05.pdf

Resolução no 3, de 20 de junho de 2014. Institui Diretrizes Curriculares Nacionais do Curso de Graduação em Medicina e dá outras providências. Recuperado em 4 de julho de 2018 http://portal.mec. gov.br/index.php?option=com_docman\&view=download\&alias=15874-rces003-14\&category_ slug=junho-2014-pdf\&Itemid $=30192$

Resolução no 5, de 15 de março de 2011. Institui as Diretrizes Curriculares Nacionais para os cursos de graduação em Psicologia, estabelecendo normas para o projeto pedagógico complementar para a Formação de Professores de Psicologia. Recuperado em 4 de julho de 2018 http://portal.mec.gov.br/ index.php?option=com_docman\&view=download\&alias=7692-rces005-11-pdf\&Itemid=30192

Shakespeare, T., \& Kleine, I. (2013). Educating health professionals about disability: a review of interventions. Health and Social Care Education, 2(2), 20-37.

Shakespeare, T., Iezzoni, L. I., \& Groce, N. E. (2009). Disability and the training of health professionals. The Lancet, 374(9704), 1815-1816. DOI: https://doi.org/10.1016/S0140-6736(09)62050-X

Sousa, E. M. de, \& Almeida, M. A. P. T. (2017). Atendimento ao surdo na atenção básica: perspectiva da equipe multidisciplinar. Id on Line Revista Multidisciplinar e de Psicologia, 10(33), 72-82. DOI: https://doi.org/10.14295/idonline.v10i33.589 
Souza, M. T. de, \& Porrozzi, R. (2017). Ensino de libras para os profissionais de saúde: uma necessidade premente. Revista Práxis, 1(2), 1-4.

Sullivan, A., \& Mendonca, R. (2017). Impact of a Fieldwork Experience on Attitudes Toward People with Intellectual Disabilities. American Journal of Occupational Therapy, 71(6). DOI: 7106230010p1$7106230010 \mathrm{p} 8$

Symons, A. B., Morley, C. P., McGuigan, D., \& Akl, E. A. (2014). A curriculum on care for people with disabilities: effects on medical student self-reported attitudes and comfort level. Disability and Health Journal, 7(1), 88-95. DOI: 10.1016/j.dhjo.2013.08.006

Trollor, J. N., Ruffell, B., Tracy, J., Torr, J. J., Durvasula, S., Iacono, T., ... Lennox, N. (2016). Intellectual disability health content within medical curriculum: an audit of what our future doctors are taught. BMC medical education, 16(1), 105.

Washington Institute of Medicine. (2007). The future of Disability in America. Washington, DC: National Academies Press. Recuperado em 4 de abril de 2017 de http://www.nationalacademies.org/hmd/ / media/Files/Report\%20Files/2007/The-Future-of-Disability-in-America/Disabilityreportbrief.pdf

World Health Organization. (2011). Word report on disability. Malta: WHO.

Recebido em: 27/08/2019

Reformulado em: 22/11/2019

Aceito em: 02/01/2020 
ASSUNÇÃO, M.L.B. et al. 


\section{Errata}

No artigo "Atendimento em Saúde à Pessoa com Deficiência e a Formação Inicial do Profissional de Saúde: o que Há rntre Nós?"

"Health Care for the Person with Disability and the Initial Training of the Health Professional: what Is Between Us?"

Onde se lê: http://dx.doi.org/10.1590/s1413-65382620000100008

Leia-se: https://doi.org/10.1590/1980-54702020v26e0181 